\title{
Do changes in STEC diagnostics mislead interpretation of disease surveillance data in Switzerland? Time trends in positivity, 2007 to 2016
}

Fabienne Beatrice Fischer ${ }^{1,2}$, Apolline Saucy ${ }^{1,2}$, Claudia Schmutz ${ }^{1,2}$, Daniel Mäusezahl1,2

1. Swiss Tropical and Public Health Institute, Basel, Switzerland

2. University of Basel, Basel, Switzerland

Correspondence: Daniel Mäusezahl (daniel.maeusezahl@unibas.ch)

Citation style for this article:

Fischer Fabienne Beatrice, Saucy Apolline, Schmutz Claudia, Mäusezahl Daniel. Do changes in STEC diagnostics mislead interpretation of disease surveillance data in Switzerland? Time trends in positivity, 2007 to 2016. Euro Surveill. 2020;25(33):pii=1900584. https://doi.org/10.2807/1560-7917.ES.2020.25.33.1900584

Article submitted on 19 Sep 2019 / accepted on 22 Apr 2020 / published on 20 Aug 2020

Background: Laboratory-confirmed cases of Shiga toxin-producing Escherichia coli (STEC) have been notifiable to the National Notification System for Infectious Diseases in Switzerland since 1999. Since 2015, a large increase in case numbers has been observed. Around the same time, syndromic multiplex PCR started to replace other diagnostic methods in standard laboratory practice for gastrointestinal pathogen testing, suggesting that the increase in notified cases is due to a change in test practices and numbers. Aim: This study examined the impact of changes in diagnostic methods, in particular the introduction of multiplex PCR panels, on routine STEC surveillance data in Switzerland. Methods: We analysed routine laboratory data from 11 laboratories, which reported $61.9 \%$ of all STEC cases from 2007 to 2016 to calculate the positivity, i.e. the rate of the number of positive STEC tests divided by the total number of tests performed. Results: The introduction of multiplex PCR had a strong impact on STEC test frequency and identified cases, with the number of tests performed increasing sevenfold from 2007 to 2016. Still, age- and sex-standardised positivity increased from $0.8 \%$ in 2007 to $1.7 \%$ in 2016. Conclusion: Increasing positivity suggests that the increase in case notifications cannot be attributed to an increase in test numbers alone. Therefore, we cannot exclude a real epidemiological trend for the observed increase. Modernising the notification system to address current gaps in information availability, e.g. diagnostic methods, and improved triangulation of clinical presentation, diagnostic and serotype information are needed to deal with emerging disease and technological advances.

\section{Introduction}

Infections caused by Shiga toxin (Stx)producing Escherichia coli (STEC) are generally mild and self-limiting or even asymptomatic. However, particularly in children and elderly people, STEC infections can lead to severe gastroenteritis with haemorrhagic diarrhoea and life-threatening conditions, e.g. haemolytic uraemic syndrome (HUS) $[1,2]$.

STEC transmission can occur through the consumption of contaminated food and drinks, or by direct contact with infected individuals or animals shedding the bacterium* $\left.{ }^{*}, 3-5\right]$. STEC infections are endemic in Europe, including Switzerland $[6,7]$. Cases occur sporadically or in outbreaks; a large outbreak attributed to contaminated sprouts occurred in Germany in 2011 [8]. Smaller outbreaks have also been reported, e.g. there was an outbreak in Italy in 2013 and in Romania in 2016, both were suspected to be caused by contaminated dairy products $[9,10]$. Considering 22 years of populationbased data up to 2012, Majowicz et al. estimated in 2014 that STEC leads to an estimated 2.8 million illness cases per year, including 3,800 cases of HUS, globally [11].

The National Notification System for Infectious Diseases (NNSID) of the Swiss Federal Office of Public Health (FOPH) has been receiving all notifications of laboratory-confirmed STEC infections since 1999. Case numbers were generally constant until 2010, with only a few laboratories reporting STEC cases in Switzerland. An increase in cases was observed in 2011 following the outbreak in Germany, before returning to expected yearly fluctuations, and then markedly increasing since 2015 [12]. Given that this increase was observed around the same time as the introduction of syndromic multiplex PCR panels for stool analyses in standard laboratory practice in Switzerland [12], it was hypothesised that these panels were the cause of the increase in notified STEC cases. Traditionally, routine testing of stool samples for bacterial pathogens involved only $C$ ampylobacter spp., Salmonella spp. and Shigella spp. using culture-based techniques. With syndromic multiplex PCR panels, stool samples can be tested for 


\section{FIGURE 1}

Number of STEC notifications to NNSID versus number of positive STEC tests of 11 diagnostic laboratories, and total number of STEC notifications to NNSID per year, Switzerland, 2007-2016

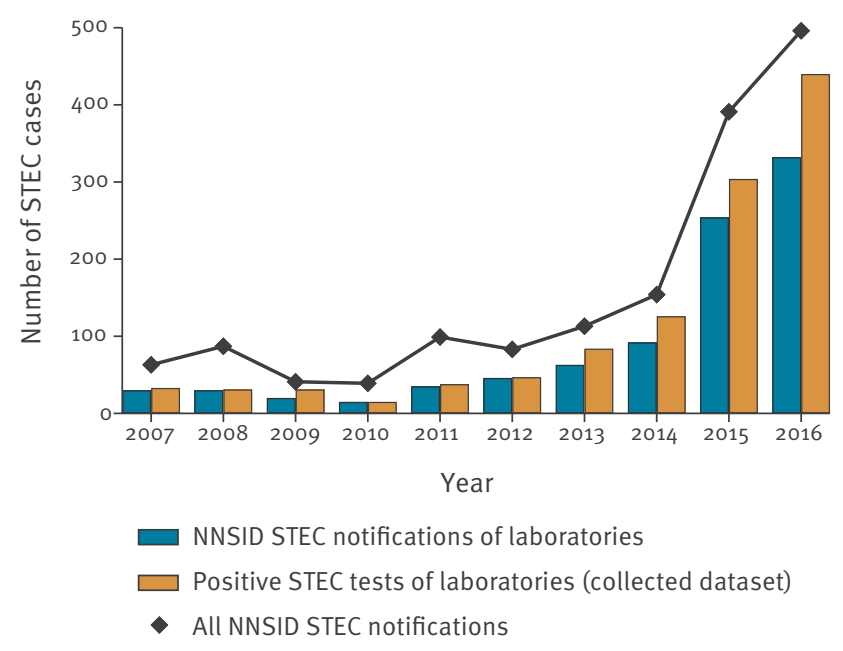

NNSID: National Notification System for Infectious Diseases; STEC: Shiga toxin-producing Escherichia coli.

up to 22 pathogens, including STEC, in one single run $[12,13]$.

Prior to the gradual introduction of multiplex PCR to the routine diagnostics between 2014 and 2015, STEC was only specifically tested for in Switzerland upon physician request, and this rarely happened. Current testing practice includes the use of small syndromic enteric bacterial panels for testing in patients without a travel history or a larger gastrointestinal panel if travel history is reported on the test order form [7].

A qualitative assessment found that Swiss laboratory experts uniformly agreed that the increase in STEC case numbers was due to the introduction and increasing use of multiplex PCR panels [7]. We set out to conduct a quantitative investigation as to whether an increase in the STEC testing rate associated with the use of the panels is what led to the increased notification of cases.

Our study assesses the development of the STEC positivity in the Swiss population between 2007 and 2016 using routine laboratory data, and gives insight into the epidemiology and notification numbers of STEC infections in Switzerland.

\section{Methods}

The study uses pre-existing records from the routine work of diagnostic laboratories. Swiss regulatory authorities report 106 authorised or accredited diagnostic laboratories, but not all of them perform STEC diagnostics [14]. Therefore and for feasibility reasons, we decided in 2016 to purposively select 11 diagnostic laboratories to be included in our study. First, the laboratories with the most STEC notifications the year before were selected and their coverage of Swiss regions was checked. For underrepresented regions, we added the top reporting laboratories of these regions to the sample. Our final sample included all regions of Switzerland, and both hospital and private diagnostic laboratories. The organisation of infectious disease diagnostics in Switzerland does not allow for estimating the population covered by the laboratories.

Anonymised, individual-based testing data on STEC from the laboratories' pre-existing records were received from the $\mathrm{FOPH}$. Data collected comprised all tests performed for STEC between January 2007 and December 2016, including positive and negative test outcomes. Our resulting database included date of test, test result, test method, patient identification number, and patients' date of birth, sex and canton of residence.

Test records indicating a patient resided outside of Switzerland and those without a conclusive test result were omitted. Duplicate entries, defined as identical values for all variables, and repeated tests were excluded from the analyses. Repeated tests were defined as more than one test performed for the same patient during a single disease episode.

The analysis was planned a priori and was performed using STATA version 14.0 (StataCorp, Texas, United States (US)). A statistical significance level of alpha 0.05 was chosen for all tests and models.

We use the term positivity as the rate of number of positive tests to the total number of tests performed for STEC $[15,16]$. Positivity was calculated for different demographic groups, test methods, spatial (i.e. patients' canton of residence) and temporal (annual and seasonal) trends. The main outcome, annual positivity, was age- and sex-adjusted using direct standardisation with the sample population (2007-2016) as reference population.

We calculated odds ratios (ORs) for the association between test result and test year, test month, season, a discrete time trend variable, sex, age group, laboratory, test method and greater region using univariable logistic regression. Season was modelled using a sine and cosine function with an annual period. The time trend was a discrete variable constructed of all test months combining the test month and test year variables. The greater regions correspond to the seven regions of Switzerland as specified by the Nomenclature of Territorial Units for Statistics (NUTS)2. Categories with most observations were chosen as reference categories, except for the seasonality (first month of the year).

We defined a multivariable mixed-effect logistic regression model a priori, independent of the outcome of the univariable regression, to calculate adjusted ORS (aORs). The model's explanatory variables included 


\section{FIGURE 2}

Total number of STEC tests performed and number of positive tests by test method (A) and by laboratory (B), 11 diagnostic laboratories, Switzerland, 2007-2016

\section{A. Number of tests performed and positives by methods}

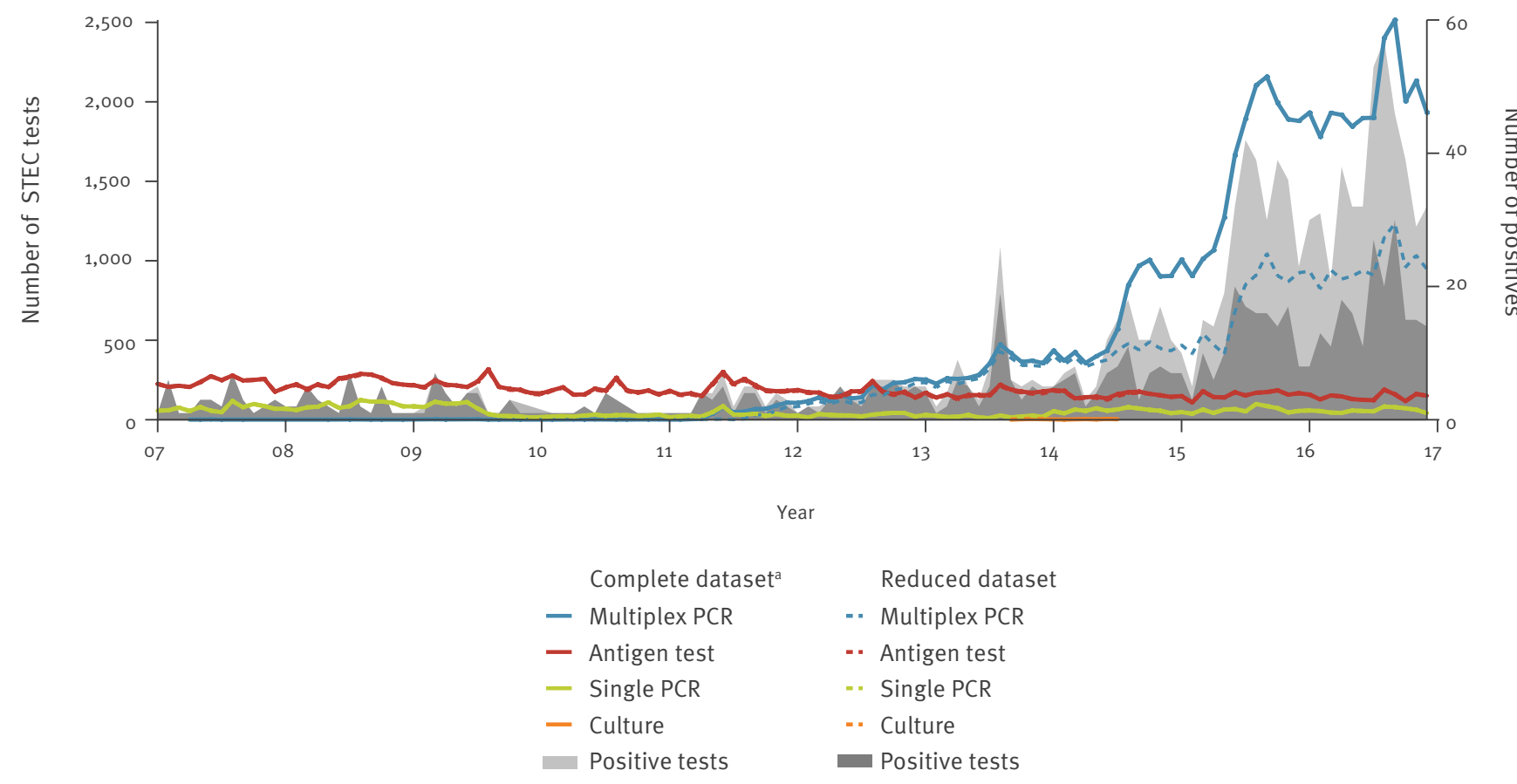

B. Number of tests performed and positives by methods and laboratory
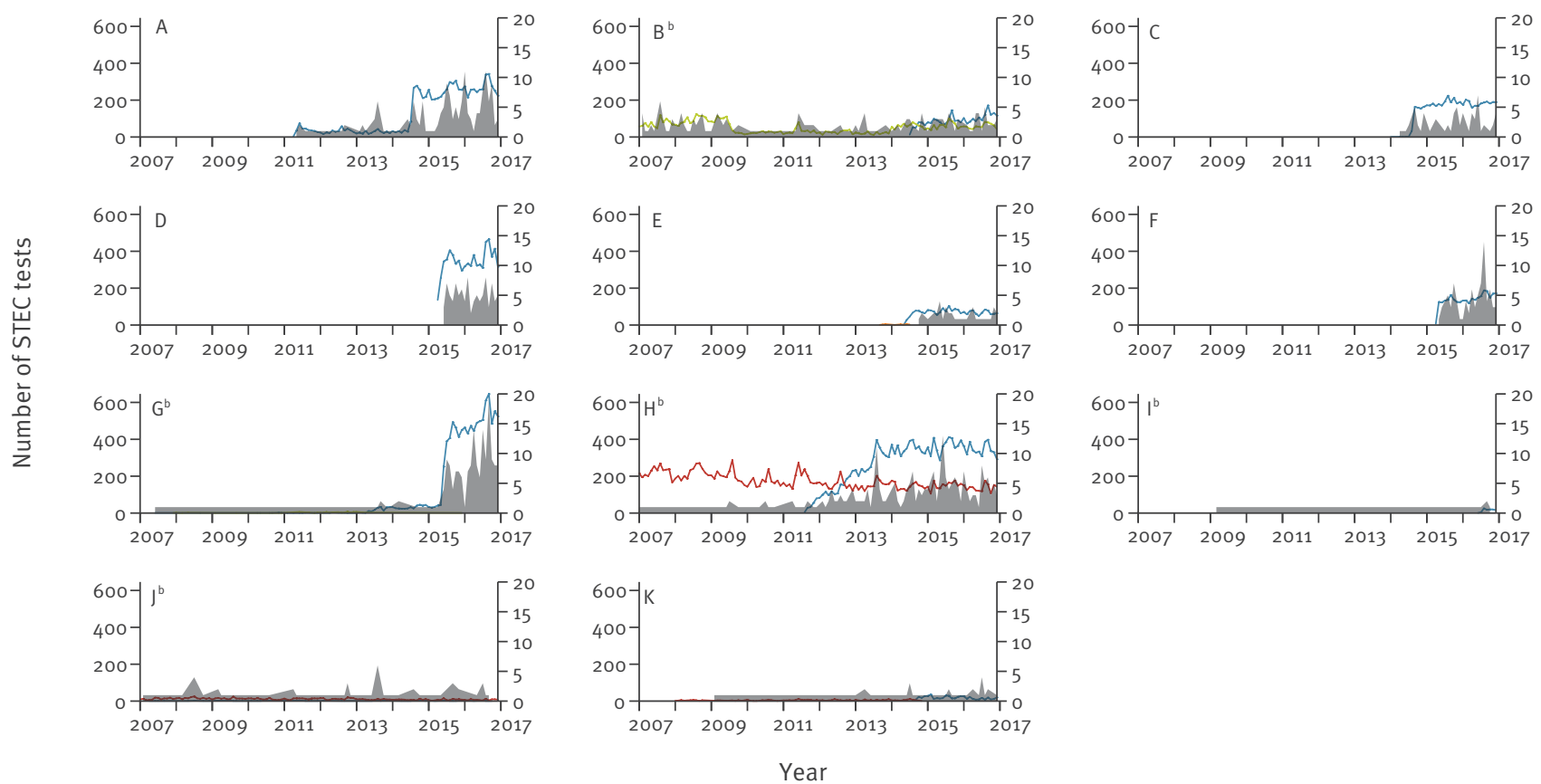

STEC: Shiga toxin-producing Escherichia coli.

${ }^{a}$ Complete dataset refers to data from all 11 laboratories, while reduced dataset refers to only the five laboratories providing data for the entire study period.

${ }^{\mathrm{b}}$ The five laboratories providing data for the entire study period. For laboratories $\mathrm{G}$ and I, the numbers starting at 2007 are too small to appear on the figure. 


\section{FIGURE 3}

Age- and sex-standardised positivity of STEC testing, 11 diagnostic laboratories, Switzerland, 2007-2016

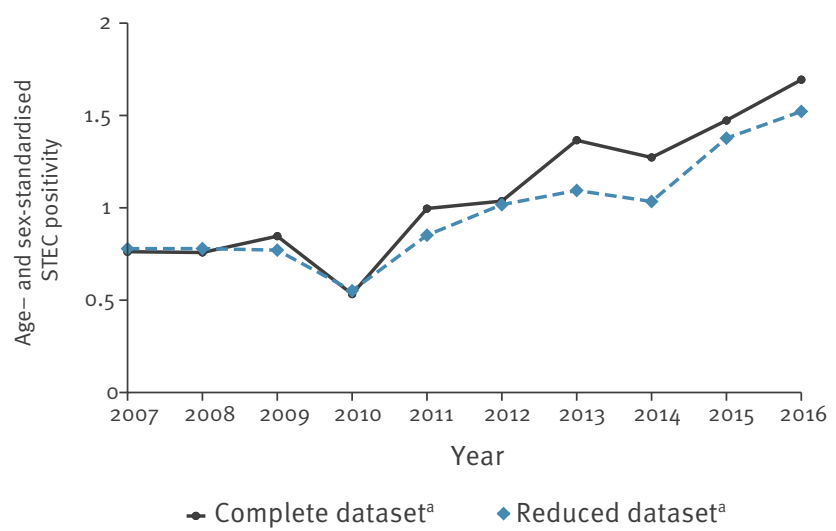

STEC: Shiga toxin-producing Escherichia coli.

a Complete dataset refers to data from all 11 laboratories, while reduced dataset refers to only the five laboratories providing data for the entire study period.

sex, age group, seasonality, time trend, greater region, diagnostic test method, and an interaction term for sex and age group. Laboratories were included as a random effect variable to account for clustering. Clustering on patient level (same identification number) was omitted.

Finally, we compared the fully adjusted multivariable model to a multivariable model without adjustment for test method in order to validate the results and ensure the consistency of the time trend, independently from the diagnostic method.

Based on multivariable regression results, we computed predicted probabilities for a positive test result, and plotted them for direct visualisation and comparison of categories and models.

We also performed a sensitivity analysis, omitting laboratories not providing data for the entire study period to account for the impact of the missing data. For relevant figures, both the complete dataset referring to data from all 11 laboratories, and the reduced dataset, referring to only the laboratories providing data for the entire study period, are shown.

\section{Ethical statement}

The study was conducted under the Epidemics Act (SR 818.101). The study team received anonymised laboratory data from the $\mathrm{FOPH}$, who had received alreadyanonymised data directly from the laboratories. Other data (notification data, population statistics) are publicly available from the FOPH or the Swiss Federal Statistical Office.

\section{FIGURE 4}

STEC positivity by laboratory, nine diagnostic laboratories ${ }^{\text {a }}$ Switzerland, 2007-2016

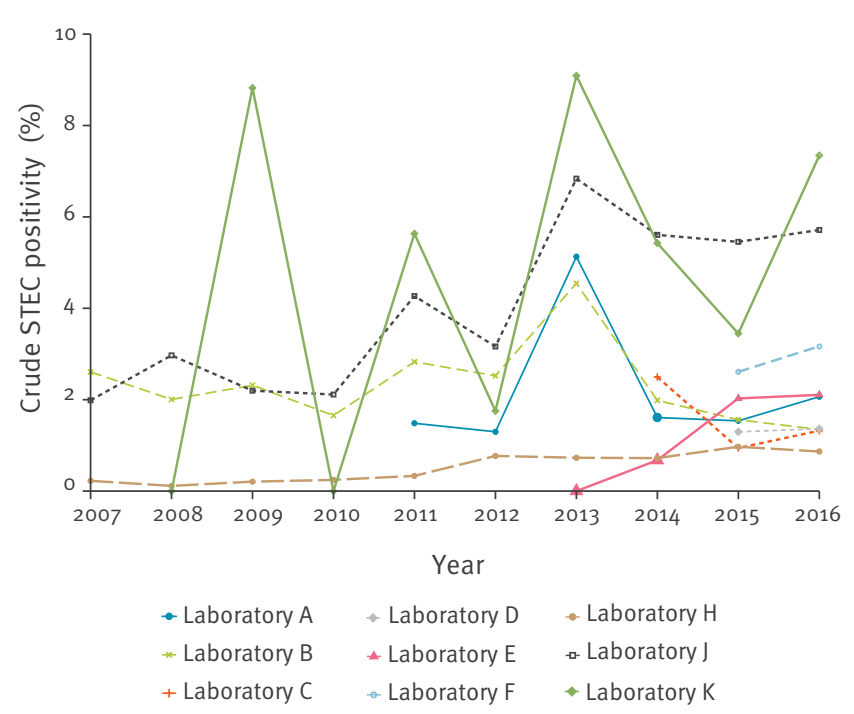

STEC: Shiga toxin-producing Escherichia coli.

a Two of the 11 laboratories comprising the dataset are not shown because of the large fluctuations in positivity (range: $0-50 \%$ ) because of small testing numbers.

\section{Results}

Number of test records and STEC-positives

The 11 participating laboratories provided 91,685 STEC test records, of which, 1,366 were positives. Five laboratories (laboratories $\mathrm{B}, \mathrm{G}, \mathrm{H}, \mathrm{I}$ and $\mathrm{J}$ ) provided data for the entire study period of 2007 to $2016(n=61,916)$. Three laboratories (C, D and F) started performing STEC testing between 2014 and 2015 with the introduction of multiplex PCR panels, two laboratories ( $A$ and E) could not extract all data requested because of changes in their data storage system and one laboratory (K) did not specify a reason for missing years of data. Sensitivity analyses omitting laboratories not providing data for the entire study period showed that observed trends were robust. Therefore, the complete dataset without omission is presented and discussed. Relevant figures show the data with and without omission.

Following our exclusion criteria, 1,407 records, including 22 positives, were excluded. Further, 71 records (3 positives) with missing sex or age, 1,110 duplicated entries (31 positives) and 3,054 repeated tests (96 positives) were excluded. The final dataset comprised 86,043 records, of which, 1,149 were positives.

Figure 1 shows the number of notified STEC cases in the NNSID and in our dataset. In concert, the laboratories selected for this study reported $61.9 \%$ of all cases registered in the NNSID between 2007 and 2016 (range $39.4 \%$ in 2011 to $73.2 \%$ in 2009 ). 
TABLE A

Odds ratios for a positive STEC test result of the uni- and multivariable logistic regression models, Switzerland, $2007-2016(\mathrm{n}=86,043)$

\begin{tabular}{|c|c|c|c|c|c|}
\hline Variable & $\mathrm{n}$ & OR & $95 \% \mathrm{Cl}$ & $\mathrm{aOR}^{\mathrm{a}}$ & $95 \% \mathrm{Cl}$ \\
\hline \multicolumn{6}{|l|}{ Age group (year) } \\
\hline Under 1 & 2,915 & 0.97 & $0.67-1.40$ & 1.28 & $0.72-2.28$ \\
\hline $1-4$ & 8,855 & $1.88^{\mathrm{b}}$ & $1.56-2.27$ & $3.38^{\mathrm{b}}$ & $2.56-4.45$ \\
\hline $5-9$ & 2,593 & $1.80^{\mathrm{b}}$ & $1.34-2.43$ & $1.66^{\mathrm{c}}$ & $1.07-2.58$ \\
\hline $10-19$ & 5,898 & 1.03 & $0.79-1.35$ & 1.03 & $0.71-1.49$ \\
\hline $20-39$ & 21,971 & Ref & NA & Ref & NA \\
\hline $40-59$ & 19,404 & 1.00 & $0.84-1.20$ & 1.03 & $0.81-1.31$ \\
\hline $60-79$ & 17,685 & 1.10 & $0.92-1.32$ & 1.05 & $0.82-1.34$ \\
\hline Over 79 & 6,722 & 1.14 & $0.89-1.45$ & 1.11 & $0.81-1.52$ \\
\hline \multicolumn{6}{|l|}{ Sex } \\
\hline Male & 38,209 & 1.03 & $0.91-1.16$ & 0.93 & $0.72-1.20$ \\
\hline Female & 47,834 & Ref & NA & Ref & NA \\
\hline \multicolumn{6}{|c|}{ Male, age group (year) } \\
\hline Under 1 & 1,582 & NA & NA & 1.14 & $0.52-2.47$ \\
\hline $1-4$ & 4,962 & NA & NA & 0.92 & $0.62-1.36$ \\
\hline $5-9$ & 1,325 & NA & NA & 1.23 & $0.67-2.27$ \\
\hline $10-19$ & 2,827 & NA & NA & 1.14 & $0.66-1.95$ \\
\hline $20-39$ & 9,080 & NA & NA & Ref & NA \\
\hline $40-59$ & 8,833 & NA & NA & 1.02 & $0.70-1.47$ \\
\hline $60-79$ & 7,408 & NA & NA & 1.27 & $0.88-1.84$ \\
\hline Over 79 & 2,192 & NA & NA & 1.17 & $0.69-1.95$ \\
\hline \multicolumn{6}{|l|}{ Greater region } \\
\hline Lake Geneva region & 15,526 & $0.79^{d}$ & $0.66-0.93$ & 1.20 & $0.89-1.60$ \\
\hline Espace Mittelland & 20,000 & Ref & NA & Ref & NA \\
\hline $\begin{array}{l}\text { Northwestern } \\
\text { Switzerland }\end{array}$ & 15,273 & $0.39^{\mathrm{b}}$ & $0.32-0.49$ & $0.69^{d}$ & $0.53-0.89$ \\
\hline Zurich & 14,439 & $0.79^{d}$ & $0.66-0.94$ & $0.75^{\mathrm{c}}$ & $0.58-0.98$ \\
\hline $\begin{array}{l}\text { Eastern } \\
\text { Switzerland }\end{array}$ & 6,474 & $0.70^{d}$ & $0.55-0.90$ & 0.88 & $0.67-1.16$ \\
\hline Central Switzerland & 10,015 & 0.90 & $0.74-1.09$ & 0.92 & $0.70-1.21$ \\
\hline Ticino & 1,008 & 0.74 & $0.43-1.30$ & 1.30 & $0.73-2.32$ \\
\hline \multicolumn{6}{|l|}{ Test method } \\
\hline Multiplex PCR & 57,168 & Ref & NA & Ref & NA \\
\hline Antigen test & 22,588 & $0.37^{\mathrm{b}}$ & $0.31-0.45$ & $0.34^{\mathrm{b}}$ & $0.26-0.44$ \\
\hline Single PCR & 6,247 & $1.56^{\mathrm{b}}$ & $1.31-1.86$ & $2.31^{\mathrm{b}}$ & $1.55-3.45$ \\
\hline Culture & 24 & NC & NC & NC & NC \\
\hline
\end{tabular}

aOR: adjusted odds ratio; $\mathrm{Cl}$ : confidence interval; NA: not applicable; NC: not calculated; OR: odds ratio; Ref: reference group for comparison; STEC: Shiga toxin-producing Escherichia coli.

adjusted for sex, age group, method, temporal trend and seasonality (refer to Supplement S1 and Supplementary Figure S1 for details). Interaction between age and sex. Random effect of laboratory.

b $\mathrm{p}<0.001$.

${ }^{c} p<0.05$.

${ }^{d} p<0.01$.

e The estimates for culture-based tests could not be calculated because of small testing numbers.
Characteristics of the tested and STEC-positive population

Median age of the tested population increased significantly from 30 to 43 years between 2007 and 2016 (test for trend: $\mathrm{p}<0.01$, Supplementary Table S1). The proportion of females tested in this period was $55.6 \%$ on average and remained level throughout the test years. The median age of the tested population differed significantly between laboratories (KruskalWallis test: $p<0.01$, range: $27-55$, overall median: 40; data not shown) and greater regions (Kruskal-Wallis test: $p<0.01$, range: 37-44; data not shown).

Similarly, among the STEC-positive population, the median age increased significantly from 2007 to 2016, while the proportion of females remained stable (test for trend: $p$ <0.01, Supplementary Table S1). Median age differed significantly between laboratories (KruskalWallis test: $p<0.01$, range: $2.5-55$, overall median: 36 ; data not shown), but not between regions (KruskalWallis test: $p=0.399$, range: $34-68$; data not shown). The average number of disease episodes per person was one, with a maximum of four for 122 persons (data not shown).

\section{Laboratories, diagnostic methods and greater regions}

The variables laboratory, greater region and test method were strongly correlated (see Supplementary Figure S2).

The diagnostic methods performed included multiplex PCR $(66.5 \%, n=57,168)$, antigen test $(26.3 \%$, $n=22,588)$, single PCR, i.e. PCR panels targeting STEC/ pathogenic $E$. coli only $(7.3 \%, n=6,247)$, and culturebased diagnostics ( $<0.1 \%, n=24)$. Sixteen ( $(0.1 \%)$ tests did not have a test method specified (outsourced tests). Multiplex PCR panels used were mainly BD MAX (normal or extended) Enteric Bacterial Panel (BD, Franklin Lakes, US) (51.6\%), xTAG Gastrointestinal Pathogen Panel (Luminex, Austin, US) (36.1\%), BioFire FilmArray Gastrointestinal Panel (BioFire, Salt Lake City, US) (5.9\%) and Seegene, not specified whether Allplex Gastrointestinal Panel or Seeplex Diarrhoea ACE Detection (Seegene, Seoul, South Korea) (4.6\%). All available information on the test methods applied as reported by the laboratories is presented in Supplementary Table S2.

The number of tests performed using the antigen test, single PCR or culture remained stable between 2007 and 2016, while the number of multiplex PCR panels performed increased by $42 \%$ (Figure $2 \mathrm{~A}$ ). The five laboratories providing data for the entire study period were using single PCR or antigen tests before the introduction of multiplex PCR (Figure 2B). Only one of these five laboratories continued using primarily antigen tests for the entire study period. 


\section{TABLE B}

Odds ratios for a positive STEC test result of the uni- and multivariable logistic regression models, Switzerland, 2007-2016 $(\mathrm{n}=86,043)$

\begin{tabular}{|c|c|c|c|c|c|}
\hline Variable & $\mathrm{n}$ & OR & $95 \% \mathrm{Cl}$ & $\mathrm{aOR}^{\mathrm{a}}$ & $95 \% \mathrm{Cl}$ \\
\hline Time trend & 86,043 & $1.00 \mathrm{~b}$ & $1.00-1.01$ & $1.00 \mathrm{C}$ & $1.00-1.01$ \\
\hline \multicolumn{6}{|l|}{ Test month } \\
\hline January & 6,040 & $0.50^{b}$ & $0.37-0.68$ & NA & NA \\
\hline February & 5,529 & $0.59^{d}$ & $0.44-0.80$ & NA & NA \\
\hline March & 6,137 & $0.58^{b}$ & $0.43-0.77$ & NA & NA \\
\hline April & 5,872 & $0.76^{c}$ & $0.58-0.99$ & NA & NA \\
\hline May & 6,357 & $0.69^{d}$ & $0.53-0.90$ & NA & NA \\
\hline June & 7,084 & $0.77^{c}$ & $0.60-0.99$ & NA & NA \\
\hline July & 7,321 & 1.08 & $0.86-1.35$ & NA & NA \\
\hline August & 9,154 & Ref & NA & NA & NA \\
\hline September & 8,919 & $0.68^{d}$ & $0.54-0.87$ & NA & NA \\
\hline October & 8,098 & $0.78^{c}$ & $0.61-0.99$ & NA & NA \\
\hline November & 8,000 & $0.71^{d}$ & $0.55-0.91$ & NA & NA \\
\hline December & 7,532 & $0.62^{b}$ & $0.47-0.81$ & NA & NA \\
\hline \multicolumn{6}{|l|}{ Seasonality } \\
\hline $\sin \left(\left(d^{\star} 2^{\star} \pi\right) / T\right)$ & 86,043 & $0.84^{b}$ & $0.77-0.91$ & $0.89^{b}$ & $0.82-0.98$ \\
\hline $\cos \left(\left(d^{\star} 2^{\star} \pi\right) / T\right)$ & 86,043 & $0.83^{b}$ & $0.76-0.90$ & $0.81^{\mathrm{c}}$ & $0.75-0.89$ \\
\hline \multicolumn{6}{|l|}{ Test year } \\
\hline 2007 & 3,711 & $0.53^{d}$ & $0.37-0.76$ & NA & NA \\
\hline 2008 & 3,978 & $0.47^{b}$ & $0.32-0.67$ & NA & NA \\
\hline 2009 & 3,421 & 0.54 & $0.38-0.79$ & NA & NA \\
\hline 2010 & 2,536 & $0.35^{\mathrm{b}}$ & $0.21-0.59$ & NA & NA \\
\hline 2011 & 3,393 & $0.67^{c}$ & $0.48-0.94$ & NA & NA \\
\hline 2012 & 4,483 & $0.63^{d}$ & $0.47-0.85$ & NA & NA \\
\hline 2013 & 6,152 & 0.82 & $0.65-1.04$ & NA & NA \\
\hline 2014 & 10,246 & $0.74^{d}$ & $0.61-0.90$ & NA & NA \\
\hline 2015 & 21,484 & $0.85^{c}$ & $0.74-0.99$ & NA & NA \\
\hline 2016 & 26,639 & Ref & NA & NA & NA \\
\hline \multicolumn{6}{|l|}{ Laboratory } \\
\hline$A$ & 8,712 & $2.98^{b}$ & $2.44-3.64$ & NA & NA \\
\hline B & 8,861 & $3.15^{\mathrm{b}}$ & $2.59-3.83$ & NA & NA \\
\hline C & 5,102 & $2.09^{b}$ & $1.60-2.75$ & NA & NA \\
\hline D & 7,181 & $2.13^{\mathrm{b}}$ & $1.68-2.70$ & NA & NA \\
\hline$E$ & 2,197 & $2.84^{b}$ & $2.02-4.00$ & NA & NA \\
\hline $\mathrm{F}$ & 2,904 & $4.80^{b}$ & $3.75-6.16$ & NA & NA \\
\hline G & 9,852 & $2.86^{b}$ & $2.36-3.48$ & NA & NA \\
\hline $\mathrm{H}$ & 38,796 & Ref & NA & NA & NA \\
\hline 1 & 121 & $9.66^{\mathrm{b}}$ & $4.46-20.94$ & NA & NA \\
\hline$J$ & 1,438 & $6.14^{\mathrm{b}}$ & $4.55-8.28$ & NA & NA \\
\hline K & 879 & $8.09^{b}$ & $5.81-11.27$ & NA & NA \\
\hline
\end{tabular}

aOR: adjusted odds ratio; $\mathrm{Cl}$ : confidence interval; NA: not applicable; NC: not calculated; OR: odds ratio; Ref: reference group for comparison; STEC: Shiga toxin-producing Escherichia coli.

a Adjusted for sex, age group, method, temporal trend and seasonality (refer to Supplement S1 and Supplementary Figure S1 for details). Interaction between age and sex. Random effect of laboratory.

${ }^{b} p<0.001$.

${ }^{c} p<0.05$.

${ }^{\mathrm{d}} \mathrm{p}<0.01$.

e The estimates for culture-based tests could not be calculated because of small testing numbers.

\section{Positivity}

The number of tests for STEC increased sevenfold from 2007 to $2016(3,711$ to 26,639$)$ while the number of positive test results increased 13 -fold ( 33 to 440 ). The age- and sex-standardised positivity of STEC testing increased from $0.8 \%$ in 2007 to $1.7 \%$ in 2016 (Figure 3).

Positivity increased for all age categories. The positivity calculated over the entire study period was highest for children aged $1-4$ years $(192 / 8,855,2.2 \%)$ and increased from $1.4 \%(11 / 809)$ in 2007 to $2.9 \%$ $(51 / 1,734)$ in 2016 . The largest relative increase was in individuals $\geq 80$ years of age, from no case among 146 in 2007 to $1.8 \%(45 / 2,449)$ in 2016.

The overall positivity is similar for men $(518 / 38,209$, $1.4 \%)$ and women $(631 / 47,834,1.3 \%)$ and increased from $0.6(11 / 1,705)$ and $1.1 \%(22 / 2,006)$ to $1.7 \%$ $(198 / 11,682)$ and $1.6 \%(242 / 14,957)$, respectively, from 2007 to 2016.

The positivity and trend in positivity differed across laboratories (Figure 4). The overall positivity ranged from $0.6 \%(245 / 38,796)$ to $5.8 \%(7 / 121)$. There were large fluctuations in positivity for some laboratories because of small testing numbers.

Positivity further differed by test method. We did not calculate the positivity of culture-based tests because there were few observations and because of our exclusion process for repeated tests (observations excluded if used as confirmation tests). The positivity across all test years was highest for tests using single PCR $(147 / 6,247,2.4 \%)$ and lowest for the antigen test (129/22,588, 0.6\%); positivity of multiplex PCR panels was at $1.5 \%(870 / 57,168)$. The positivity of multiplex PCR increased from $1.1 \%(80 / 7,617)$ in 2014 to $1.7 \%(418 / 24,190)$ in 2016 . In contrast, the positivity of single PCR and antigen tests started to decrease in 2014 and 2015 respectively, after PCR peaking at $4.3 \%$ $(11 / 256)$ in 2013 and antigen tests at $1.4 \%(27 / 1,896)$ in 2014 .

\section{Predictors of a positive diagnostic test result}

The univariable regressions showed a marginal but significant trend for the time trend variable (OR: 1.003, p 0.01 , Table). All test years except 2013 showed decreased odds for a positive test outcome compared with the reference year 2016. All calendar months except July have smaller odds for a positive test outcome than the reference month August.

The age groups 1 to 4 years and 5 to 9 years were almost twice as likely to have a positive test outcome (OR 1.88, p<0.001 and OR 1.80, p<0.001) than the reference category 20 to 39 years. No difference was observed between sexes.

Compared with multiplex PCR panels, the use of the antigen test had a $63 \%$ lower probability to generate a positive test outcome (OR $0.37, p<0.001$ ), while the 


\section{FIGURE 5}

Predicted probability for a positive STEC test outcome for the fully adjusted multivariable model and the model excluding adjustment for test method for the complete (A) and reduced (B) dataset, 11 diagnostic laboratories, Switzerland, 2007-2016

\section{A. Complete dataset ${ }^{\mathrm{a}}$}

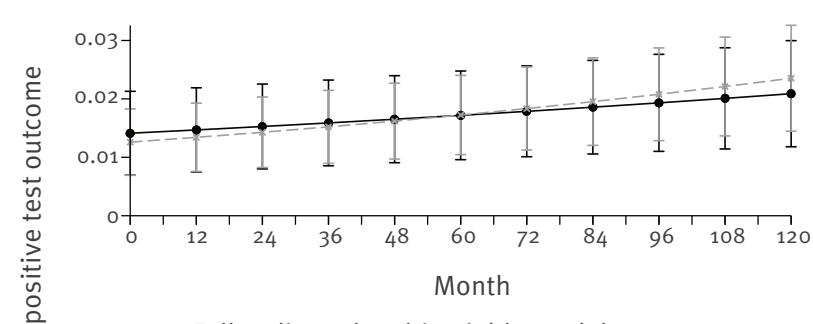

- Fully adjusted multivariable model

* Model without adjustment for diagnostic method

\section{B. Reduced dataset ${ }^{\mathrm{a}}$}

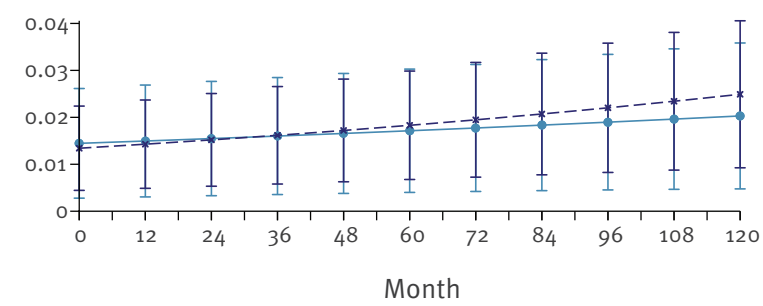

- Fully adjusted multivariable model

* Model without adjustment for diagnostic method

STEC: Shiga toxin-producing Escherichia coli.

${ }^{a}$ Complete dataset refers to data from all 11 laboratories, while reduced dataset refers to only the five laboratories providing data for the entire study period.

use of single PCR showed $56 \%$ higher chance for a positive test outcome (OR 1.56, p<0.001).

The ORs and significance levels from the fully adjusted multivariable model, presented in the Table, varied only marginally from the univariable models and do not alter the interpretation; therefore, they are not commented here.

Predicted probabilities based on the fully adjusted multivariable model showed an increasing time trend for all test methods and regions.

Comparison of the fully adjusted multivariable model to a multivariable model excluding the adjustment for test method showed increasing predicted probabilities for both models, but with a smaller slope for the fully adjusted model (Figure 5).

\section{Discussion}

We investigated the apparent epidemic increase of STEC infections seen in the rise of case notifications in the Swiss NNSID. We calculated positivity as the rate of all positive diagnostic STEC tests to the total number of STEC tests performed. The 11 laboratories in our study reported almost two-thirds (61.9\%) of all STEC cases in the NNSID between 2007 and 2016. Positivity increased since 2007.

Culture-independent diagnostic tests for STEC The increase of STEC cases in Switzerland coincides with the introduction of multiplex PCR panels as a new diagnostic method for STEC detection. The impact of changes in diagnostic approaches on public health surveillance has been highlighted before, especially concerning the switch from culture-dependent to culture-independent diagnostics for food-borne diseases [17-19]. This switch is particularly important for STEC, as the case definitions for STEC in the European Union/ European Economic Area (EU/EEA) and Switzerland are not limited to culture-confirmed cases, but include the detection of the Stx1 or Stx2 antigen or their respective genes [20]. Increases in STEC notifications in Ireland were explained by the shift from culture-dependent to culture-independent diagnostic methods; the latter showing higher sensitivity and ability to detect non0157 STEC $[21,22]$.

The 11 Swiss diagnostic laboratories included in our study switched to culture-independent methods for STEC detection before 2007; hence, the impact thereof cannot be assessed using our data.

\section{Considerations when using multiplex PCR} panels for STEC diagnosis

The introduction of multiplex PCR panels for gastrointestinal pathogens is the next paradigm shift in diagnostics for food-borne diseases after switching to culture-independent tests.

In most of our study laboratories, the use of multiplex PCR panels as routine diagnostic methods was introduced between 2011 and 2015. Since then, multiplex panels comprise the largest proportion of all diagnostic tests performed for STEC and have led to an increase in test numbers. The increase in test volume, resulting in more positives notified, originates from a larger proportion of the population being automatically screened for STEC. This screening happens for two reasons: (i) the testing for a specific gastrointestinal pathogen, e.g. Campylobacter spp., now also implicitly leads to a STEC test or (ii) the physician orders a gastrointestinal panel when the patient presents with diarrhoea, i.e. syndromic testing. Previously, a test for STEC was predominantly ordered if the patient was a child and/ or reported a bloody stool and/or reported a history of travel because of higher probabilities to develop severe complications such as HUS [23-25]. We hypothesised that if the increase in new STEC cases was a result of the introduction of multiplex PCR only (leading to less targeted screening) there would be a decrease in positivity because of a lower pre-test probability for a positive test outcome. But this decrease in positivity is not reflected in our data. Instead, the increase in STEC cases is disproportionally higher compared with the increase in test volume, resulting in the observed increase in positivity. 
Part of the increased testing could also stem from a change in physicians' test-ordering behaviour following the raising of public awareness for STEC infections. However, laboratory experts reported that tests specifically for STEC are rarely ordered by treating physicians [7]. Therefore, STEC tends to largely be an unintentional finding and its clinical relevance for the individual patient may be arguable. Questions on reporting to the patient and appropriate treatment, see Davis et al. [26], and mandatory notification still need to be addressed.

Furthermore, using multiplex PCR increases the number of cases found because of the higher sensitivity of PCR compared with other conventional diagnostic methods, and the increased probability of detecting co-infections [27-30]. A study among staff members of meat-processing companies in Switzerland found 3.5\% asymptomatic carriers of STEC [31]. Assuming a similar prevalence of asymptomatic carriers in the general population and the possibility that such asymptomatic STEC carriers become infected with another diarrhoeagenic pathogen, multiplex PCR would detect both the symptom-causing pathogen and the asymptomatic STEC co-infection.

While it is clear that changes in the diagnostic landscape can influence surveillance data and trend monitoring, we believe that this change only explains part of the increase in STEC case notifications in Switzerland.

From our analyses, indications for a real increase in STEC incidence independent of the diagnostic test method are threefold: (i) Our logistic regressions and predicted probabilities for a positive STEC test outcome showed an increasing trend between 2007 and 2016 even after adjusting for the diagnostic method, (ii) the predicted probabilities for a positive STEC test show an increasing trend for all methods (multiplex PCR, single $P C R$ and antigen test) and (iii) an increase in positivity was also seen in two laboratories introducing multiplex PCR panels late, i.e. in the second half of 2016 , or not at all. Based on these three findings, we argue that the increase in notified STEC cases is a combination of changing test practices and a real increase in incidence of STEC infections among the Swiss population.

\section{Rising incidence of STEC infections}

Age and sex distributions of STEC patients in Switzerland remained unchanged since the observation period 2007 to 2016. We conclude that the observed incidence increase is independent of potential changes in STEC risk groups.

If our findings suggest a true increase in STEC, the epidemiology of HUS also needs to be considered. In Switzerland, the number of HUS cases remained relatively constant from 1999 to 2015 in terms of absolute numbers; hence, there was a relative decrease of HUS among notified STEC cases [12]. Thus, the increase in STEC notifications observed is likely to represent mainly mild cases and/or asymptomatic co-infections that might have been present but undetected in the past.

We propose that a changing distribution of STEC serogroups among cases could be an explanation for the change in disease severity. In other studies, 0157 STEC cases were found to mostly be associated with the development of severe disease, i.e. HUS, although the importance of non-0157 infections as a cause for HUS is being increasingly recognised [32-34].

STEC culture and subsequent analysis of isolates are not routinely performed in Switzerland; the proportion of culture-based tests in our raw dataset of routinely conducted tests in 11 laboratories was only $0.1 \%$ (78/89,081, raw dataset). The scarce information on serotype distribution primarily comes from studies published by the Swiss National Reference Centre for Enteropathogenic Bacteria and Listeria (NENT) $[35,36]$. Analysing 2017 data, Nüesch-Inderbinen et al. [36] indicated that an isolate for further characterisation could be successfully obtained from less than $30 \%$ of multiplex PCR positive samples, suggesting limited information on serotypes in Switzerland compared with other countries. Still, using these studies and the results from research in similar contexts abroad, we can discuss the epidemiology of rising STEC incidence within Switzerland.

The two studies out of NENT reported a decrease in the proportion of STEC stx2 carrying and eae carrying variants, which are both associated with severe disease in Switzerland $[35,36]$. Over the course of several years, the proportion of non-0157 STEC associated with human disease increased in Switzerland, other European countries and the US [35,37,38]. On the other hand, a 2013 study found that healthy people can shed stx-carrying bacteriophages that might lead to $s t x$-positive multiplex PCR test results [39].

No EU/EEA country reported an increase in STEC notification numbers to the extent observed in Switzerland (eightfold increase, 2012-2016), except Romania, where 1 case was reported in 2012 while 29 were found in 2016 following an intensified testing after a HUS outbreak [38]. In Finland, the increase in reported cases between 2012 and 2016 was fourfold, with multiplex PCR screening introduced in $2013[38,40]$. In Norway, the notification rate increased from 0.6 to 7.6 per 100,000 population between 2007 and 2017, noting that this increase occurred mostly after 2014 and coinciding with the introduction of multiplex PCR diagnostics [41].

STEC patients associated with a recent outbreak in Finland were classified as rather mild cases [42]. The increasing STEC notifications in Norway were associated with an increasing proportion of cases classified as low-virulent while case numbers of HUS were generally constant [41]. The US also reported an 
increased incidence of STEC cases in 2017 compared with 2014 to 2016 , although not to the extent observed in Switzerland [37]. Further, the incidence of HUS in children in the US remained similar in 2016 compared with 2013-2015, while non-0157 infections increased, resulting in a relative decrease of 0157 cases. This again supports the hypothesis of an association between disease severity and serogroup, with a trend of culture-independent diagnostic tests increasing detection of less virulent strains.

Information on co-infections is neither available from the notification system nor from the data collected by the laboratories. However, up to $10 \%$ of the STEC strains obtained from clinical samples of ill individuals and identified by Nüesch-Inderbinen et al. were the same as strains isolated from the faecal samples of healthy individuals suggesting that not the identified STEC, but another pathogen was causing the symptoms [36]. This is in line with earlier reports that 3.5\% of meat factory workers were asymptomatic STEC carriers [31]. In Norway, co-infections were observed in 15\% of notified STEC cases detected using multiplex PCR [41]. Hence, it is likely that a minor but relevant proportion of the newly identified infections by multiplex PCR are asymptomatic co-infections.

\section{Implications of changing disease patterns on} STEC surveillance in Switzerland

Current disease surveillance for STEC in Switzerland neither is designed to account for changes in diagnostics nor systematically distinguish between strains (particularly $0_{157}$ and non-0157) that could reflect differences in virulence.

From a health systems perspective, monitoring the usage of diagnostic methods and testing algorithms applied for each notifiable pathogen among authorised and accredited diagnostic laboratories could complement surveillance data.

Since the implementation of a revised Epidemics Act in Switzerland in 2016, diagnostic laboratories are required to report the number of tests conducted for certain notifiable diseases (but excluding STEC) to the FOPH once a year. This annual reporting of summary statistics was established in the hope of improving interpretation of routine surveillance data through the incorporation of denominator data similar to that here in our study; without the need to mandate resourceintensive research for each pathogen. However, analyses of these summary statistics indicate that data quality is rather poor and that too many factors play a role to conclude on reasons for changes in test and case numbers based on summary statistics [7].

The increase of STEC cases, which are mostly mild, and the shift in serotype distribution as shown by others, changes the interpretation of STEC notifications as clinical and public health relevance needs to be considered. We believe it is critical that all cases of STEC infections, regardless of clinical relevance, are reported in order to identify clusters and sources and thus support outbreak control. However, the current effectiveness of the Swiss surveillance system for STEC could be improved incorporating strain typing information that would guide intervention and control measures, yet this also depends on achieving higher success rates of STEC isolation after PCR-positive results. The federal public health authorities recognise the need to modernise the current notification system toward electronic reporting which addresses the current issues of information availability, including more information on the diagnostic test methods used, and data inconsistency, ensuring more harmonisation between laboratory-based notifications of test results with clinical information obtained from physicians' mandatory notifications (personal communication, Daniel Koch (FOPH), August 2019).

\section{Limitations}

First, we selected our sample of 11 laboratories based on their contribution to the latest NNSID notifications. This choice favoured laboratories that had switched to multiplex PCR and may therefore not be representative of all laboratories in Switzerland. However, we adjusted for test method in our main trend analysis, thereby accounting for bias towards an over-representation of multiplex PCR. Second, our study only uses the actual information available to the laboratories; clinical information could not be obtained. Third, as partly evident from the data, culture-based tests and typing of STEC was very rarely performed by the participating laboratories; hence, microbiological data were not available for analysis. However, analysis of pre-existing (routine) data from laboratories can support the evaluation of surveillance data in a time- and resource-efficient manner, which could potentially be harnessed for other pathogens. Fourth, we noted that in recent years, NNSID case numbers differed from the number of positive test results recorded in the laboratories' individual datasets. This means that positive cases were either under-reported to the NNSID, or the NNSID excluded certain reports from their official statistics or the number of positive test results in our sample was overestimated because of, for example, an insufficient exclusion of repeated tests. Finally, the correlation of laboratory, greater region and test method hampered the evaluation of spatial trends. Differences in testing and positivity rates between greater regions in Switzerland largely depend on the laboratories chosen. The differences can either relate to true differences in tests ordered by physicians between regions or they could be because the laboratories selected for our sample under-, over- or misrepresent the laboratories within their region.

\section{Conclusion}

Since 2015, the notifications for STEC markedly increased in Switzerland. Meaningful interpretation of such surveillance data requires that every aspect of the disease trajectory, from changes in awareness 
(among physicians and patients) and testing behaviour to the choice of diagnostic method, are taken into consideration.

STEC surveillance has been heavily impacted by recent changes in diagnostic methods given the lack of culture-based confirmative testing and previously infrequent, but targeted testing for STEC. The switch from targeted STEC testing to co-testing of virtually all stool samples submitted for basic stool bacteriology using multiplex PCR panels has notably increased the test volume for STEC in Switzerland. However, we have found a rise in STEC cases that is disproportionally high compared to the increase in test volume, suggesting that there has been a real increase in STEC infection incidence in Switzerland.

The recently observed changes in the frequency of different serogroups and the stability of HUS cases suggests that the trend observed for STEC is mostly attributable to rather mild cases. Surveillance systems should be adapted to include information on diagnostic methods used considering the rapid development of new laboratory techniques. Modernising the notification system should also allow for a better triangulation of notified information on clinical presentation, diagnostic approaches and serotypes, provided the success rate of isolating multiplex PCR-positive samples increases.

\section{${ }^{\star}$ Erratum}

A mistake in the pathogen description was corrected on 24 August 2020.

\section{Acknowledgements}

The authors thank Christian Schindler (Swiss Tropical and Public Health Institute) for statistical advice, Adrian Egli (University Hospital Basel) for feedback on repeated testing and Angelika Fruth (Robert Koch Institute) for sharing experience on STEC surveillance in Germany. Roger Stephan (Institute for Food Safety and Hygiene, University of Zurich) provided feedback on the manuscript. Various staff of the Swiss Federal Office of Public Health (FOPH) provided detailed insights to the Swiss surveillance system and information on the notification data; we appreciate the contributions made by Daniel Koch and Mirjam MäusezahlFeuz, Department of Communicable Diseases, FOPH. The authors much appreciate the support of the following laboratories providing data for the study: ADMed Microbiologie / Reto Lienhard (La Chaux-de-Fonds), Analytica Medizinische Laboratorien AG (Zurich), Bioanalytica (Lucerne), Dianalabs (Geneva), Laboratoire de bactériologie des HUG / Jacques Schrenzel (Geneva), IFIK / Sara Droz (Bern), MCL Medizinische Laboratorien (Niederwangen), Labor Synlab / André Burnens and Marcel Brandenberger (Lucerne), Viollier AG (Allschwil) and two other Swiss diagnostic laboratories.

Funding statement: This study was funded by the Swiss Federal Office of Public Health (FOPH). The FOPH provided the framework of the study which was carried out under the Epidemics Act (SR 818.101). FOPH were not involved in the data processing, analysis and interpretation of the results.
Conflict of interest

None declared.

\section{Authors' contributions}

CS and DM conceived and designed the study. Data collection and processing was performed by AS, with FBF and CS. FBF conducted the analysis. FBF, AS, CS and DM interpreted the results. FBF and AS wrote the first draft of the manuscript. All authors contributed to the revisions of the manuscript and approved the final version.

\section{References}

1. World Health Organization (WHO). Fact sheet. E. coli. Geneva: WHO. [Accessed 12 Oct 2018]. Available from: http://www.who. int/en/news-room/fact-sheets/detail/e-coli

2. Tarr PI, Gordon CA, Chandler WL. Shiga-toxin-producing Escherichia coli and haemolytic uraemic syndrome. Lancet. 2005;365(9464):1073-86. https://doi.org/10.1016/S01406736(05)71144-2 PMID: 15781103

3. Chart H. Are all infections with Escherichia coli 0157 associated with cattle? Lancet. 1998;352(9133):1005. https:// doi.org/10.1016/S0140-6736(05)60072-4 PMID: 9759740

4. Grif K, Orth D, Lederer I, Berghold C, Roedl S, Mache CJ, et al. Importance of environmental transmission in cases of EHEC 0157 causing hemolytic uremic syndrome. Eur J Clin Microbiol Infect Dis. 2005;24(4):268-71. https://doi.org/10.1007/ S10096-005-1320-Z PMID: 15902533

5. Vernozy-Rozand C. Detection of Escherichia coli $\mathrm{O}_{157}: \mathrm{H} 7$ and other verocytotoxin-producing E. coli (VTEC) in food. J Appl Microbiol. 1997;82(5):537-51. https://doi. org/10.1111/j.1365-2672.1997.tb03584.x PMID: 9172396

6. European Centre for Disease Prevention and Control (ECDC). Shiga-toxin/verocytotoxin-producing Escherichia coli (STEC) VTEC) infection. In: ECDC. Annual epidemiological report for 2018. Stockholm: ECDC; 2020. Available from: https://www. ecdc.europa.eu/sites/default/files/documents/shiga-toxinverocytototoxin-escherichia-coli-annual-epidemiologicalreport-2018.pdf

7. Schmutz C. Foodborne diseases in Switzerland: Understanding the burden of illness pyramid to improve Swiss infectious disease surveillance [dissertation]. Basel, Switzerland: University of Basel, Faculty of Science; 2018.

8. Buchholz U, Bernard H, Werber D, Böhmer MM, Remschmidt C, Wilking $\mathrm{H}$, et al. German outbreak of Escherichia coli $\mathrm{O}_{104}: \mathrm{H}_{4}$ associated with sprouts. N Engl J Med. 2011;365(19):1763-70. https://doi.org/10.1056/NEJMoa1106482 PMID: 22029753

9. Germinario C, Caprioli A, Giordano M, Chironna M, Gallone MS, Tafuri S, et al., all participants of the Outbreak investigation team. Community-wide outbreak of haemolytic uraemic syndrome associated with Shiga toxin 2-producing Escherichia coli $\mathrm{O}_{26: \mathrm{H} 11}$ in southern Italy, summer 2013. Euro Surveill. 2016;21(38):30343. https://doi.org/10.2807/1560-7917. ES.2016.21.38.30343 PMID: 27684204

10. Usein C-R, Ciontea AS, Militaru CM, Condei M, Dinu S, Oprea $M$, et al. Molecular characterisation of human Shiga toxinproducing Escherichia coli 026 strains: results of an outbreak investigation, Romania, February to August 2016. Euro Surveill. 2017;22(47):17-00148. https://doi.org/10.2807/1560-7917. ES.2017.22.47.17-00148 PMID: 29183554

11. Majowicz SE, Scallan E, Jones-Bitton A, Sargeant JM, Stapleton J, Angulo FJ, et al. Global incidence of human Shiga toxinproducing Escherichia coli infections and deaths: a systematic review and knowledge synthesis. Foodborne Pathog Dis. 2014;11(6):447-55. https://doi.org/10.1089/fpd.2013.1704 PMID: 24750096

12. Hächler H, Stephan R. Auffälliger Anstieg der Meldezahlen enterohämorrhagischer E. coli-Infektionen über die letzten Monate in der Schweiz: Einfluss neuer Multiplex PCRMethoden in der Primär-Diagnostik? [Striking increase in the number of reports of enterohaemorrhagic $E$. coli infections over the last months in Switzerland: Influence of new multiplex PCR methods in primary diagnostics?]. BAG Bulletin.2015;52:988-90.German.

13. Binnicker MJ. Multiplex molecular panels for diagnosis of gastrointestinal infection: Performance, result interpretation, and cost-effectiveness. J Clin Microbiol. 2015;53(12):3723-8. https://doi.org/10.1128/JCM.02103-15 PMID: 26311866

14. Swissmedic.Laboratory establishment licences issued under the old process. Bern: Swissmedic. [Accessed 24 Jan 2019]. 
Available from: https://www.swissmedic.ch/swissmedic/ en/home/humanarzneimittel/bewilligungen zertifikate/ microbiological-laboratories/bewilligungsinhaber.html

15. Schmutz C, Burki D, Frei R, Mäusezahl-Feuz M, Mäusezahl D. Testing for Chlamydia trachomatis: time trends in positivity rates in the canton of Basel-Stadt, Switzerland. Epidemiol Infect. 2013;141(9):1953-64. https://doi.org/10.1017/ So950268812002567 PMID: 23158540

16. Bless PJ, Schmutz C, Sartori K, Mäusezahl D. Time trends of positivity rates from foodborne pathogen testing in Switzerland, 2003 to 2012. Swiss Med Wkly. 2017;147:w14569. PMID: 29282700

17. Kehl SC. Role of the laboratory in the diagnosis of enterohemorrhagic Escherichia coli infections. J Clin Microbiol. 2002;40(8):2711-5. https://doi.org/10.1128/JCM.40.8.27112715.2002 PMID: 12149318

18. Cronquist AB, Mody RK, Atkinson R, Besser J, Tobin D'Angelo $M$, Hurd $S$, et al. Impacts of culture-independent diagnostic practices on public health surveillance for bacterial enteric pathogens. Clin Infect Dis. 2012;54(Suppl 5):S432-9. https:// doi.org/10.1093/cid/cis267 PMID: 22572666

19. Moran-Gilad J. How do advanced diagnostics support public health policy development? Euro Surveill. 2019;24(4):1900068. https://doi.org/10.2807/1560-7917.ES.2019.24.4.1900068 PMID: 30696524

20. European Commission, Directorate-General for Health and Food Safety. Commission Implementing Decision (EU) 2018/945 of 22 June 2018 on the communicable diseases and related special health issues to be covered by epidemiological surveillance as well as relevant case definitions. Official Journal of the European Union. Luxembourg: Publications Office of the European Union. 6.7.2018:L 170. Available from: https://eur-lex.europa.eu/eli/dec_impl/2018/945/oj

21. Johnson RP, Clarke RC, Wilson JB, Read SC, Rahn K, Renwick $\mathrm{SA}$, et al. Growing concerns and recent outbreaks involving non-0157:H7 serotypes of verotoxigenic Escherichia coli. J Food Prot. 1996;59(10):1112-22. https://doi.org/10.4315/0362028X-59.10.1112 PMID: 31195470

22. Rice T, Quinn N, Sleator RD, Lucey B. Changing diagnostic methods and increased detection of verotoxigenic Escherichia coli, Ireland. Emerg Infect Dis. 2016;22(9):1656-7. https://doi. org/10.3201/eid2209.160477 PMID: 27322897

23. Clogher P, Hurd S, Hoefer D, Hadler JL, Pasutti L, Cosgrove $\mathrm{S}$, et al. Assessment of physician knowledge and practices concerning Shiga toxin-producing Escherichia coli infection and enteric illness, 2009, Foodborne Diseases Active Surveillance Network (FoodNet). Clin Infect Dis. 2012;54(Suppl 5):S446-52. https://doi.org/10.1093/cid/cis246 PMID: 22572668

24. Rivas M, Chinen I, Miliwebsky E, Masana M. Risk factors for Shiga toxin-producing Escherichia coli-associated human diseases. Microbiol Spectr. 2014;2(5). https://doi.org/10.1128/ microbiolspec.EHEC-0002-2013 PMID: 26104362

25. Bless PJ, Muela Ribera J, Schmutz C, Zeller A, Mäusezahl D. Acute gastroenteritis and campylobacteriosis in Swiss primary care: The viewpoint of general practitioners. PLoS One. 2016;11(9):e0161650. https://doi.org/10.1371/journal. pone.0161650 PMID: 27603141

26. Davis TK, Van De Kar NCAJ, Tarr PI. Shiga toxin/verocytotoxinproducing Escherichia coli infections: Practical clinical perspectives. Microbiol Spectr. 2014;2(4):0025-2014. PMID: 26104210

27. Khare R, Espy MJ, Cebelinski E, Boxrud D, Sloan LM, Cunningham SA, et al. Comparative evaluation of two commercial multiplex panels for detection of gastrointestinal pathogens by use of clinical stool specimens. J Clin Microbiol. 2014;52(10):3667-73. https://doi.org/10.1128/JCM.01637-14 PMID: 25100818

28. Buss SN, Leber A, Chapin K, Fey PD, Bankowski MJ, Jones $\mathrm{MK}$, et al. Multicenter evaluation of the BioFire FilmArray gastrointestinal panel for etiologic diagnosis of infectious gastroenteritis. J Clin Microbiol. 2015;53(3):915-25. https:// doi.org/10.1128/JCM.02674-14 PMID: 25588652

29. Stockmann C, Rogatcheva M, Harrel B, Vaughn M, Crisp $R$, Poritz $M$, et al. How well does physician selection of microbiologic tests identify Clostridium difficile and other pathogens in paediatric diarrhoea? Insights using multiplex PCR-based detection. Clin Microbiol Infect. 2015;21(2):179.e915. https://doi.org/10.1016/j.cmi.2014.07.011 PMID: 25599941

30. Harrington SM, Buchan BW, Doern C, Fader R, Ferraro MJ, Pillai DR, et al. Multicenter evaluation of the BD max enteric bacterial panel PCR assay for rapid detection of Salmonella spp., Shigella spp., Campylobacter spp. (C. jejuni and C. coli), and Shiga toxin 1 and 2 genes. J Clin Microbiol. 2015;53(5):1639-47. https://doi.org/10.1128/JCM.03480-14 PMID: 25740779
31. Stephan R, Ragettli S, Untermann F. Prevalence and characteristics of verotoxin-producing Escherichia coli (VTEC) in stool samples from asymptomatic human carriers working in the meat processing industry in Switzerland. J Appl Microbiol. 2000;88(2):335-41. https://doi.org/10.1046/j.13652672.2000.00965.x PMID: 10736003

32. Käppeli U, Hächler H, Giezendanner N, Beutin L, Stephan R. Human infections with non-0157 Shiga toxin-producing Escherichia coli, Switzerland, 2000-2009. Emerg Infect Dis. 2011;17(2):180-5. https://doi.org/10.3201/eid1702.100909 PMID: 21291586

33. Kuehne A, Bouwknegt M, Havelaar A, Gilsdorf A, Hoyer P, Stark $\mathrm{K}$, et al. Estimating true incidence of $\mathrm{O}_{157}$ and non-O157 Shiga toxin-producing Escherichia coli illness in Germany based on notification data of haemolytic uraemic syndrome. Epidemiol Infect. 2016;144(15):3305-15. https://doi.org/10.1017/ So950268816001436 PMID: 27468812

34. Freedman SB, Xie J, Neufeld MS, Hamilton WL, Hartling L, Tarr PI, et al. Shiga toxin-producing Escherichia coli infection, antibiotics, and risk of developing Hemolytic Uremic Syndrome: A meta-analysis. Clin Infect Dis. 2016;62(10):1251-8. https://doi.org/10.1093/cid/ciw099 PMID: 26917812

35. Fierz L, Cernela N, Hauser E, Nüesch-Inderbinen M, Stephan R. Characteristics of Shigatoxin-producing Escherichia coli strains isolated during 2010-2014 from human infections in Switzerland. Front Microbiol. 2017;8:1471. https://doi. org/10.3389/fmicb.2017.01471 PMID: 28824596

36. Nüesch-Inderbinen M, Morach M, Cernela N, Althaus D, Jost $M$, Mäusezahl M, et al. Serotypes and virulence profiles of Shiga toxin-producing Escherichia coli strains isolated during 2017 from human infections in Switzerland. Int J Med Microbiol. 2018;308(7):933-9. https://doi.org/10.1016/j. ijmm.2018.06.011 PMID: 30042042

37. Marder EP, Griffin PM, Cieslak PR, Dunn J, Hurd S, Jervis R, et al. Preliminary incidence and trends of infections with pathogens transmitted commonly through food - Foodborne Diseases Active Surveillance Network, 10 U.S. Sites, 20062017. MMWR Morb Mortal Wkly Rep. 2018;67(11):324-8. https://doi.org/10.15585/mmwr.mm6711a3 PMID: 29565841

38. European Centre for Disease Prevention and Control (ECDC). Shiga-toxin/verocytotoxin-producing Escherichia coli (STEC/ VTEC) infection. In: Annual Epidemiological Report for 2016. Stockholm: ECDC; 2018. [Accessed 12 Oct 2018]. Available from: https://ecdc.europa.eu/en/publications-data/shigatoxinverocytotoxin-producing-escherichia-coli-stecvtecinfection-annual

39. Martinez-Castillo A, Quirós P, Navarro F, Miró E, Muniesa $M$. Shiga toxin 2-encoding bacteriophages in human fecal samples from healthy individuals. Appl Environ Microbiol. 2013;79(16):4862-8. https://doi.org/10.1128/AEM.01158-13 PMID: 23747705

40. Antikainen J, Kantele A, Pakkanen SH, Lääveri T, Riutta J, Vaara $M$, et al. A quantitative polymerase chain reaction assay for rapid detection of 9 pathogens directly from stools of travelers with diarrhea. Clin Gastroenterol Hepatol. 2013;11(10):13001307.e3. https://doi.org/10.1016/j.cgh.2013.03.037 PMID: 23639597

41. Jenssen GR, Veneti L, Lange H, Vold L, Naseer U, Brandal LT. Implementation of multiplex PCR diagnostics for gastrointestinal pathogens linked to increase of notified Shiga toxin-producing Escherichia coli cases in Norway, 2007-2017. Eur J Clin Microbiol Infect Dis. 2019;38(4):801-9. https://doi. org/10.1007/s10096-019-03475-5 PMID: 30680573

42. Kinnula S, Hemminki K, Kotilainen H, Ruotsalainen E, Tarkka E, Salmenlinna S, et al. Outbreak of multiple strains of non-0157 Shiga toxin-producing and enteropathogenic Escherichia coli associated with rocket salad, Finland, autumn 2016. Euro Surveill. 2018;23(35):1700666. https://doi.org/10.2807/15607917.ES.2018.23.35.1700666 PMID: 30180926

\section{License, supplementary material and copyright}

This is an open-access article distributed under the terms of the Creative Commons Attribution (CC BY 4.0) Licence. You may share and adapt the material, but must give appropriate credit to the source, provide a link to the licence and indicate if changes were made.

Any supplementary material referenced in the article can be found in the online version.

This article is copyright of the authors or their affiliated institutions, 2020. 